\title{
Attempted rescue of circling mice by hair cell-specific expression (Myo7a promoter) of tmie transgene*)
}

\author{
SEOJIN PARK'1), SOYOUNG JANG'1), \\ JEONG-HAN LEE**, SUNG-HYUN KIM*, ZAE YOUNG RYOO
}

\author{
School of Life Sciences, BK21 Plus KNU creative bioresearch group, Kyungpook National University, \\ Daegu, Republic of Korea \\ *Center for Laboratory Animal Resources, Kyungpook National University, Daegu, Republic of Korea \\ **Department of Physiology, School of Medicine, University of Nevada, Reno, Nevada, USA
}

Park S., Jang S., Lee J.-H., Kim S.-H., Ryoo Z. Y.

\begin{abstract}
Attempted rescue of circling mice by hair cell-specific expression (Myo7a promoter) of tmie transgene
\end{abstract}
\section{Summary}

The spontaneous mutant circling mouse (cir/cir) is deaf and displays abnormal behavior, particularly circling and head tossing. To rescue the circling mouse phenotype, we produced transgenic mice with hair cell-specific expression of the transmembrane inner ear (tmie) gene, using the Myo7a promoter, and generated cir/cir homozygous mice carrying the transgene (cir/cir-tgMyo7a) by breeding with circling mice.

The cir/cir-tgMyo7a mice still exhibited circling behavior and were unable to swim in water unlike cir/ cir-tgCMV mice and wild-type mice. An auditory brainstem response (ABR) test demonstrated that the cir/cir-tgMyo7a mice could not respond to sound. Immunohistochemical analysis showed enhanced green fluorescent protein, a marker of tmie expression, in the inner and outer hair cells of the cir/cir-tgMyo7a mice. Hair cells and spiral ganglion neurons in the cir/cir-tgMyo7a mice were recovered, but not completely. This study demonstrates that tmie transgene expression in hair cells alone could not restore wild-type hearing and behavior in circling mice.

Keywords: circling mouse, Myo7a promoter, hair cell, tmie, rescue

At birth, the morphology of the cochlear structure in mice is immature. Maturation of the inner ear proceeds along a basal to apical gradient during the early postnatal days. Stereocilia bundles are rearranged in rows of increasing height and kinocilia, and many kinds of links disappear. Other parts of the cochlea, such as spiral ganglion neurons, the stria vascularis, and the tectorial membrane, undergo maturation. The onset of hearing in mice begins at around postnatal day 14 (6-8).

Circling mice are characterized by deafness caused by degeneration of the inner ear and have a genomic deletion region in common with the spinner mouse, which is another deafness mutant. The tmie gene, which

*) This research was supported by Basic Science Research Program through the National Research Foundation of Korea (NRF-2013R1A1A2060793) funded by the Ministry and the National Research Foundation of Korea (NRF) grant funded by the Korean government (MSIP [Ministry of Science, Information Communication Technology and Future Planning]; no. 2008-0062618). This research was supported by a grant of the Korea Health Technology R\&D Project through the Korea Health Industry Development Institute (KHIDI), funded by the Ministry of Health \& Welfare, Republic of Korea (grant number HO13C0008). This research was supported by Kyungpook National University Research Fund, 2013 (2014).

1) These authors contributed equally to this work. is part of this common deletion region, is presumed to be a causative gene for the circling mouse phenotype $(2,4,16)$. To address this hypothesis, we previously produced transgenic mice in which the tmie transgene was introduced into circling mice. Under control of the cytomegalovirus (CMV) promoter, tmie was expressed in most organs and tissues, including the inner ear. These transgenic mice recovered the wild-type phenotype, with normal hearing and balancing. These data demonstrated that tmie is a main causative gene among the deleted genes of circling mice and encodes an important protein in the inner ear (19).

The hair cells of the cochlea consist of three rows of outer hair cells and one row of inner hair cells. Stereocilia bundles on the surface of hair cells connect with neuronal synapses and are responsible for transmitting stimuli. Outer hair cells and inner hair cells have different functions in response to stimuli. Inner hair cells are the sensory receptors that are responsible for the afferent information sent to the brain, while outer hair cells provide mechanical feedback from neurons 
$(9,10,15,18)$. Since the myo7 promoter is a specific promoter for the inner ear sensory cells, we used this promoter in the trial (1).

In the circling mouse, hair cell degeneration patterns of the inner ear and expression patterns of tmie are similar. Expression of the tmie protein begins in hair cells and spreads to other parts of the organ of Corti, such as hair cell bodies and supporting cells; the expression level increases during the early postnatal days (20). During the maturation of the organ of Corti in the circling mouse, stereocilia bundles on hair cells degenerate first, followed by gradual degeneration of hair cells and spiral ganglion neurons (3).

The function of tmie is related to that of hair cells, so we hypothesized that the expression of tmie in hair cells can restore the wild-type phenotype in circling mice. In this study, we generated circling mice with hair cell- specific expression of tmie. Moreover, gene therapy is a useful technique for the treatment of deafness caused by gene mutation or deletion. However, the activity of

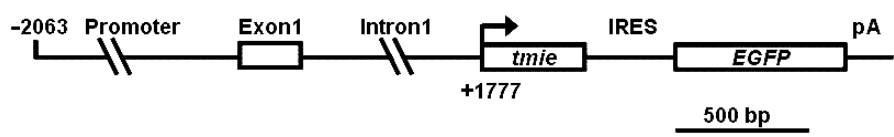

Fig. 1. Construction for overexpression of the tmie transgene. In order to induce hair cell-specific expression of mouse tmie, the Myo7A promoter was used in transgene construction. The construct contained the extending from $2 \mathrm{~kb}$ promoter sequence plus intron 1 to the Myo7a ATG initiation codon $(-2063$ to +1776$)$. The ATG initiation codon is located at position 1777. As a reporter gene, EGFP was cloned next to the tmie open reading frame (ORF), using an IRES sequence. $A$ bovine growth hormone polyA tail was added at the end of the construct.
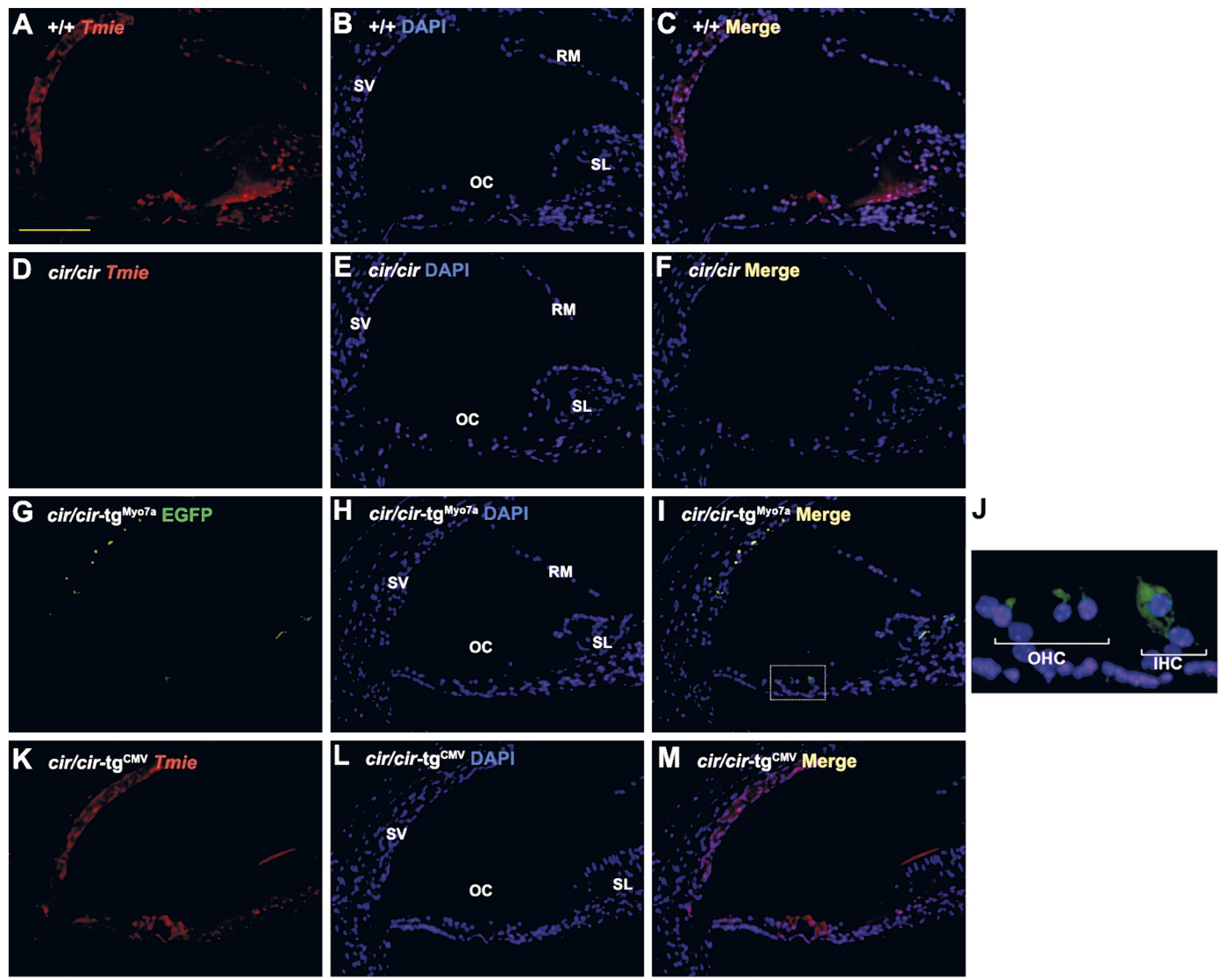

Fig. 2. Analysis of tmie expression. Staining of cross-sections from the organ of Corti in normal (+/+), circling (cir/cir), and

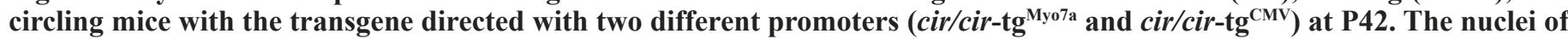
immunostained cells were counterstained with DAPI. (A-C) Tmie signals (red) are detected in the stria vascularis, organ of Corti, and spiral limbus in +/+ mice. (D-F) No positive staining is observed in the circling mouse. (G-J) The organ of Corti in cir/cir-tg mice is positive for EGFP (green, as a reporter of tmie). (J) Enlarged image of the inset from panel J. Note that the transgene is expressed only in inner and outer hair cells in the cir/cir-tg ${ }^{\mathrm{Myo} 7 \mathrm{a}}$ mouse. SV, stria vascularis; RM, Reissner's membrane; OC, organ of Corti; SL, spiral limbus; IHC, inner hair cell; OHC, outer hair cell. Scale bars (A-M): $200 \mu \mathrm{m}$. 
the gene has to occur accurately to fit the time and site. Our study will be the foundation research for therapy.

\section{Material and methods}

Generation of transgenic mice. To generate transgenic mice in which tmie is expressed in hair cells, the Myo7a promoter (1) was used for transgene construction, and an IRES sequence and enhanced green fluorescent protein (EGFP) were added to the end of the tmie open reading frame (Fig. 1). Three transgenic mice were produced by microinjection. The founder transgenic mice were mated with spontaneous mutant circling ( $\mathrm{cir} / \mathrm{cir}$ ) mice $(12,13)$. Offspring were confirmed by polymerase chain reaction. Circling mice in which tmie was expressed under the control of the CMV promoter were generated in our previous study (19).

All animal experiments were carried out in accordance with the guidelines for animal experimentation and under permission from the Institutional Animal Care and Use Committee.

Auditory brain stem response tests. Xylazine $(4 \mathrm{mg} /$ $\mathrm{kg}$ ) and ketamine $(4 \mathrm{mg} / \mathrm{kg})$ were used for anesthetization of mice, which were then tested in an electrically shielded booth. Mice were connected with needle electrodes (Grass E2 platinum) subcutaneously implanted below the tested ear (reference electrode), the vertex (active electrode), and below the contralateral ear (ground electrode).

They were stimulated by 15 -ms tonal bursts (rise-fall time $1 \mathrm{~ms}$ ) at 4, 8, 16, and $32 \mathrm{kHz}$. The sound stimuli were generated by Tucker-Davis hardware and were delivered from shielded Beyer earphones via a 13-mm tube. The Tucker Davis data acquisition system was used to generate averages (1024 epochs) of response waveforms $(1,000,000$ gain, filtered from 0.3-3.0 kHz). A 0.25-inch ACO Pacific condenser microphone (Belmont, CA, USA) was used for calibration of the sound-delivery system in a volume approximating the mouse external ear canal and expressed as the sound pressure level $(\mathrm{dB})$.

Immunohistochemical analysis of the organ of Corti. The temporal bones were fixed in $4 \%$ paraformaldehyde, decalcified with $10 \%$ ethylenediaminetetraacetic acid, dehydrated, and embedded in paraffin wax. Sections were deparaffinized and dehydrated in xylene and serial concentrations of ethanol. The immunohistochemical study was performed with an LSAB-kit Universal K680 kit, according to the manufacturer's instructions. Specimens were blocked with $3 \%$ hydrogen peroxide and $1 \%$ goat serum followed by reaction with primary antibodies, anti-tmie (19). Alexa Fluor 568-conjugated goat anti-rabbit IgG (Invitrogen) was used for detection of the primary antibody. Nuclei were stained with DAPI.

Surface preparation of the cochlea. The organ of Corti was prepared for histological analysis by first fixing the temporal bone in $4 \%$ paraformaldehyde. After fixation, the cochleae were microdissected, washed with $0.25 \%$ Triton X-100, and incubated in NF200 (Sigma) and TRITC-labeled phalloidin (Sigma). After washing, the specimens were evaluated by fluorescence microscopy.

\section{Results and discussion}

Generation of transgenic mice. In our previous study (17), we generated circling mice in which the tmie transgene was controlled by the CMV promoter ( cir/ cir-tgCMV). In the present study, we produced transgenic mice with pEGFP-N1/tmie-IRES-Myo7a and mated these with circling homozygous mice $\left(\mathrm{cir} / \mathrm{cir}^{-} \operatorname{tg}^{\mathrm{Myo}}{ }^{\mathrm{a} a}\right)$. For generation of transgenic mice, we constructed a hair cell-specific expression vector containing tmie under the control of the Myo7a (1) (Fig. 1). The Myo $7 a$ promoter has specific activity in hair cells of the inner ear. Before generating transgenic mice, we confirmed the activity of the transgene vector in vitro (data not shown).

To analyze the expression of transgenic tmie in the cochlea, we stained the cochlea from normal $(+/+)$, circling (cir/cir), cir/cir-tg ${ }^{\mathrm{CMV}}$, and $c i r / c^{2}$-tg ${ }^{\mathrm{Myo}}{ }^{\mathrm{Ma}}$ mice. The tmie protein was not detected in cir/cir mice. In the cir/cir-tg ${ }^{\text {Myo7a }}$ mice, EGFP, which served as a reporter protein of tmie expression, was detected only in the hair cells, unlike in cir/cir-tg ${ }^{\mathrm{CMV}}$ mice, in which tmie was expressed in cochlea including the organ of Corti, stria vascularis, and spiral limbus (Fig. 2).

Expression of tmie in hair cells is ineffective in rescuing circling mouse. Introduction of the tmie transgene in circling mice, restored their normal behavior and hearing function (19). However, behavioral evaluation showed that $\mathrm{cir} / \mathrm{cir}^{-\operatorname{tg}^{\mathrm{Myo}}}{ }^{\mathrm{aa}}$ mice could not swim and exhibited abnormal behavior, such as circling. When we tested the auditory brainstem response (ABR), the wild-type phenotype was not rescued in $\mathrm{cir} / \mathrm{cir}^{-\operatorname{tg}^{\mathrm{Myo}}}{ }^{\mathrm{Ma}}$ mice in contrast with $\mathrm{cir} / \mathrm{cir}^{-\operatorname{tg}^{\mathrm{CMV}}}$ mice (Fig. 3).

Morphological analysis of the structure of the inner ear showed that in cir/cir-tg ${ }^{\mathrm{CMV}}$ mice it was restored to an extent equivalent to that of wild-type mice. In cir/cir-tg ${ }^{\mathrm{Myo}}{ }^{\mathrm{a} a}$ mice, the structure of the inner ear was restored, but not sufficiently for the normal function of the inner ear (Fig. 4). Taken together, these data show

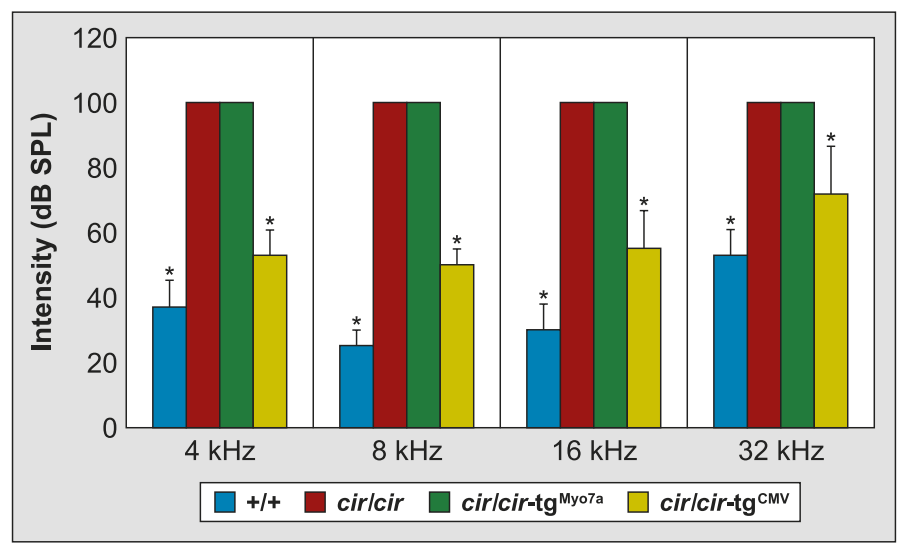

Fig. 3. Auditory brainstem responses. Mean ABR thresholds induced by tone-burst stimulation of four different groups: wild-type $(+/+)$, circling (cir/cir), and circling mice carrying the transgene directed by Myo7a and CMV promoter (cir) $c^{i r}-\operatorname{tg}^{\mathrm{My} 07 \mathrm{a}}$ and $\mathrm{cir} /$ cir-tg $^{\mathrm{CMV}}$, respectively). Average ABR thresholds in decibels referenced to sound pressure level (dB SPL) are plotted against frequency. Frequencies tested were $4,8,16$, and $32 \mathrm{kHz}$. Hearing threshold with no responses to given stimuli was referred to $100 \mathrm{~dB}$ SPL. Standard errors of the mean range are plotted for each data point. Group differences that were statistically significant are indicated by asterisks $(\mathrm{P}<\mathbf{0 . 0 5})$. 


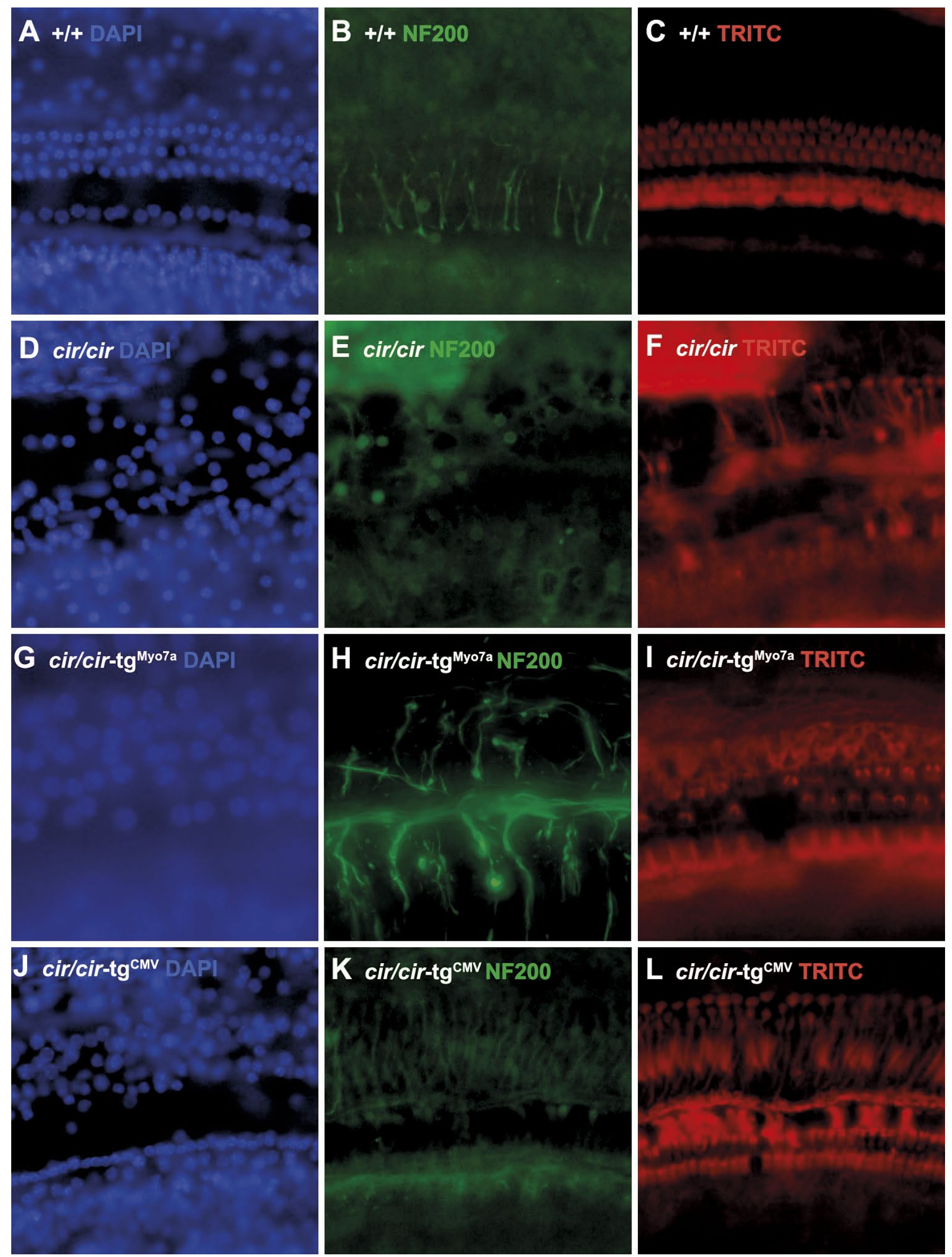

Fig. 4. Comparison of inner ear recovery. Surface preparation of the wild-type (A-C), $\operatorname{cir} /$ cir $^{(D-F)}$, cir/cir-tg $^{\mathrm{Myo} 7 \mathrm{a}}$ (G-I), and cir/cir-tgCMV (J-L). Tissues were obtained from the middle turn of the cochlea. The nuclei of immunostained cells were counterstained with DAPI (left panels). Spiral ganglion neurons were immunostained with anti-neurofilament 200 (NF200) antibodies (green, central panels). Phalloidin-TRITC was used to detect F-actin in V-shaped stereocilia bundles (red, right panels). 
that expression of tmie in hair cells could not induce a sufficient recovery of the inner ear.

Expression of tmie and degeneration of the inner ear of circling mice starts in hair cells $(3,20)$. The hair cells of circling mice do not take up gentamicin or FM1-43 (17). Accordingly, we hypothesized that circling mice have impaired mechanotransduction and that tmie is important in hair cells. We therefore produced transgenic circling mice that expressed tmie in hair cells. Our data show that expression of tmie in the hair cells could not rescue the wild-type phenotype in circling mice, in contrast with mice expressing tmie ubiquitously. In circling mice, degeneration of hair cells and spiral ganglion neurons occurs during maturation of the inner ear, whereas $c i r / c i r$-tg ${ }^{\mathrm{Myo} 7 \mathrm{a}}$ mice have normal hair cells and spiral ganglion neurons. These results demonstrate that the tmie gene is required for body balance and hearing, and it should be expressed in various parts of the inner ear, including hair cells.

We verified the effectiveness of tmie as a target of gene therapy. In our previous study (17), we produced transgenic tmie under the control of the CMV promoter and expressed it in circling mice, and these mice exhibited normal behavior and hearing. Furthermore, the expression of transgenic tmie induced the recovery of the inner ear structure. The wild-type phenotype was not rescued in cir/cir-tg ${ }^{\mathrm{Myo} 7 \mathrm{a}}$ mice. In cir/cir-tg ${ }^{\mathrm{CMV}}$ mice, the level of recovery in hearing and behavior varied depending on the tmie protein level, and the recovery of hair cells and spiral ganglion neurons depended on the transgene expression level (19). Expression of the transgene is influenced by the copy number of the integrated transgene (5), which we could not control when we used microinjection for generating transgenic mice. We suggest that the expression level of tmie in cir/cir- $\operatorname{tg}^{\text {Myo7a }}$ mice was insufficient for a detectable rescue of the wild-type phenotype.

An alternative explanation is that tmie expression is required in other parts of the inner ear, including hair cells. Recently, protein that interacts with tmie has been discovered. Tmie interacts with postcadherin 15 (PCDH15) and Lipoma HMGIC fusion partner-like 5 (LHFPL5). PCDH15 is an essential component of tip-link, which is part of the mechanotransduction machinery structure. LHFPL5 is a binding partner of PCDH15. This study suggests that tmie is an important protein in hair cells (21). However, the expression of tmie has also been found in other parts of the inner ear as well as in various tissues, such as the brain, liver, spleen, and lungs $(3,20)$. Degeneration in circling mice progresses faster than it does in other deaf mutant mice $(11,14)$. And degeneration of hair cells could not induce degeneration of spiral ganglion neurons rapidly (22), but it was followed by the loss of spiral ganglion neurons in circling mice (3).

It is possible that tmie is a necessary protein in other parts of the inner ear, including hair cells, and could have additional functions in other parts of the inner ear for normal function of the inner ear. Future studies on the function of tmie in hair cells may provide clues on the mechanotransduction mechanisms. Moreover, studies of tmie may help understand the mechanism of hearing loss in the other parts of the inner ear.

\section{References}

1. Boëda B., Weil D., Petit C.: A specific promoter of the sensory cells of the inner ear defined by transgenesis. Human Mol. Gen. 2001, 10, 1581-1589

2. Cho K. I., Lee J. W., Kim K. S., Lee E. J., Suh J. G., Lee H. J., Kim H. T., Hong S. H., Chung W. H., Chang K. T., Hyun B. H., Oh Y. S., Ryoo Z. Y.: Fine mapping of the circling (cir) gene on the distal portion of mouse chromosome 9. Comp. Med. 2003, 53, 642-648.

3. Chung W. H., Kim K. R., Cho Y. S., Cho D. Y., Woo J. H., Ryoo Z. Y., Cho K. I., Hong $S$. H.: Cochlear pathology of the circling mouse: a new mouse model of DFNB6. Acta Oto-laryngol. 2007, 127, 244-251.

4. Deol M. S., Robins M. W.: The spinner mouse. J. Heredity 1962, 53, 133-136.

5. FitzPatrick D. R.: Transcriptional consequences of autosomal trisomy: primary gene dosage with complex downstream effects. Trends in genetics: TIG 2005, 21, 249-253.

6. Forge A., Wright T.: The molecular architecture of the inner ear. Bri. Med. Bull 2002, 63, 5-24

7. Furness D. N., Richardson G. P., Russell I. J.: Stereociliary bundle morphology in organotypic cultures of the mouse cochlea. Hearing Res. 1989, 38, 95-109.

8. Groves A. K., Zhang K. D., Fekete D. M.: The genetics of hair cell development and regeneration. Ann. Rev. Neurosci. 2013, 36, 361-381.

9. Hakizimana P., Brownell W. E., Jacob S., Fridberger A.: Sound-induced length changes in outer hair cell stereocilia. Nature Comm. 2012, 3, 1094.

10. Johnson S. L., Kuhn S., Franz C., Ingham N., Furness D. N., Knipper M., Steel K. P., Adelman J. P., Holley M. C., Marcotti W.: Presynaptic maturation in auditory hair cells requires a critical period of sensory-independent spiking activity. Proc. Nat. Acad. Sci. USA 2013, 110, 8720-8725.

11. Kwon T. J., Cho H. J., Kim U. K., Lee E., Oh S. K., BokJ., Bae Y. C., Yi J. K., Lee J. W., Ryoo Z. Y., Lee S. H., Lee K. Y., Kim H. Y.: Methionine sulfoxide reductase $\mathrm{B} 3$ deficiency causes hearing loss due to stereocilia degeneration and apoptotic cell death in cochlear hair cells. Hum. Mol. Gen. 2014, 23, 1591-1601.

12. Lee J. W., Lee E. J., Hong S. H., Chung W. H., Lee H. T., Lee T. W., Lee J. R., Kim H. T., Suh J. G., Kim T. Y., Ryoo Z. Y.: Circling mouse: possible animal model for deafness. Comp. Med. 2001, 51, 550-554.

13. Lee J. W., Ryoo Z. Y., Lee E. J., Hong S. H., Chung W. H., Lee H. T., Chung K. S., Kim T. Y., Oh Y. S., Suh J. G.: Circling mouse, a spontaneous mutant in the inner ear. Experimental Animals 2002, 51, 167-171.

14. Li S., Price S. M., Cahill H., Ryugo D. K., Shen M. M., Xiang M.: Hearing loss caused by progressive degeneration of cochlear hair cells in mice deficient for the Barhl1 homeobox gene. Development 2002, 129, 3523-3532.

15. Liu H., Pecka J. L., Zhang Q., Soukup G. A., Beisel K. W., He D. Z. Characterization of transcriptomes of cochlear inner and outer hair cells. J. Neurosci. 2014, 34, 11085-11095.

16. Naz S., Giguere C. M., Kohrman D. C., Mitchem K. L., Riazuddin S., Morell R. J., Ramesh A., Srisailpathy S., Deshmukh D., Riazuddin S., Griffith A. J., Friedman T. B., Smith R. J., Wilcox E. R.: Mutations in a novel gene, TMIE, are associated with hearing loss linked to the DFNB6 locus. Am. Human Gen. 2002, 71, 632-636

17. Park S., Lee J. H., Cho H. J., Lee K. Y., Kim M. O., Yun B. W., Ryoo Z.: tmie is required for gentamicin uptake by the hair cells of mice. Comp. Med. 2013, 63, 136-142.

18. Reichenbach T., Hudspeth A. J.: The physics of hearing: fluid mechanics and the active process of the inner ear. Rep. Progress Physics Physical. Soc. 2014, 77, 076601 .

19. Shin M. J., Lee J. H., Yu D. H., Kim B. S., Kim H. J., Kim S. H., Kim M. O., Park C., Hyun B. H., Lee S., So H. S., Park R., Ryoo Z. Y.: Ectopic expression of tmie transgene induces various recovery levels of behavior and hearing ability in the circling mouse. Biochem. Biophys. Res. Comm. 2008, 374, 17-21.

20. Shin M. J., Lee J. H., Yu D. H., Kim H. J., Bae K. B., Yuh H. S., Kim M. O., Hyun B. H., Lee S., Park R., Ryoo Z. Y.: Spatiotemporal expression of tmie in the inner ear of rats during postnatal development. Comp. Med. 2010, 60, 288-294.

21. Zhao B., Wu Z., Grillet N., Yan L., Xiong W., Harkins-Perry S., Muller U.: TMIE is an essential component of the mechanotransduction machinery of cochlear hair cells. Neuron. 2014, 84, 954-967.

22. Zilberstein Y., Liberman M. C., Corfas G.: Inner hair cells are not required for survival of spiral ganglion neurons in the adult cochlea. J. Neurosci. 2012, 32 405-410

Corresponding authors: Sunghyun Kim, e-mail: shkim92@knu.ac.kr; Zae Young Ryoo, e-mail: jaewoong64@knu.ac.kr 